\title{
Perspectivas de famílias sobre as práticas de Intervenção Precoce na Infância: o que nos diz a literatura
}

\author{
Families' perspectives about the practices of Early Childhood Intervention: \\ what the literature tells us
}
Perspectivas de familias sobre las prácticas de Atención Temprana en la Infancia: lo que nos dice la literatura

\section{* Marisa Alexandra Maia Machado \\ Doutoranda na Universidade de Aveiro, Aveiro, Portugal. \\ mamm@ua.pt}

Recebido: 02 de novembro de 2017

Aprovado: 11 de fevereiro de 2019

\section{RESUMO}

As práticas centradas na família - abordagem recomendada internacionalmente para a Intervenção Precoce na Infância - atribui às famílias o papel de protagonistas no processo de apoio às crianças, através do seu envolvimento ativo, considerando as suas necessidades e preocupações. O propósito deste estudo é conhecer quais as práticas que estão a ser implementadas pelos profissionais a partir das perspectivas das famílias apoiadas. Assim, o objetivo do estudo centra-se em identificar perspectivasas práticas actuais que se encontram a ser implementadas no processo de apoio de Intervenção Precoce ao nível internacional. Com a formulação de critérios de seleção, que se basearam no ano de publicação; tipo/natureza do estudo; objetivos e sujeitos; procedemos a uma pesquisa abrangente da literatura publicada entre 2011 e 2017, existente nas bases de dados b-on, ERIC, Scopus e Scielo. Após a leitura de 1002 títulos de estudos, identificámos 79 estudos, dos quais lemos o resumo e deste passámos para a leitura integral do estudo. Pelo que encontrámos 14 estudos que preencheram os critérios de seleção e alvos de avaliação. A análise dos resultados foi guiada pelas orientações para a implementação da abordagem centrada na família e, assim, composta em quatro categorias: (1) práticas relacionais; (2) práticas participativas; (3) qualidade técnica do profissional; (4) equipa transdisciplinar. Estes estudos fornecem evidências da importância e utilidade da Intervenção Precoce na Infância e dos seus benefícios para as crianças e suas famílias. Os serviços prestados foram positivamente úteis na compreensão das necessidades e competências das famílias e das suas crianças e contribuíram para o seu desenvolvimento. Emergem contributos de indicadores de competências profissionais.

Palavras-chave: Intervenção precoce na infância; Abordagem centrada na família; Envolvimento. 


\section{ABSTRACT}

Family-centered practices - an internationally recommended practice to Early Childhood Intervention - assigns families the role of protagonists in the process of supporting children through their active engagement, taking into account their needs and concerns. The purpose of this study is to know what current practices are being adopted by professionals from the perspectives of the families supported. Thus, the objective of the study is to identify the practices that are being implemented in the process of support of Early Intervention at the international level. With the formulation of selection criteria, which were based on the year of publication; type/nature of the study; aims and subjects; we conducted a comprehensive survey of the literature published between 2011 and 2017 in the b-on, ERIC, Scopus and Scielo databases. After reading 1002 study titles, we identified 79 studies, of which we read the abstract and from this we moved on to the full reading of the study. Therefore, we found 14 studies that met the selection criteria and evaluation targets. The analysis of the results was guided by the guidelines for the implementation of the family-centered practices, and thus was composed of four categories: (1) relational practices; (2) participatory practices; (3) technical quality of the professional; (4) transdisciplinary team. These studies provide evidence of the importance and usefulness of Early Childhood Intervention and its benefits to children and their families. The services provided were positively useful in understanding the needs and skills of families and their children and contributed to their development. Contributions of indicators of professional competences emerge.

Keywords: Early childhood intervention; Family-centered practices; Engagement.

\section{RESUMEN}

Las prácticas centradas en la familia - enfoque recomendado internacionalmente para la Atención Temprana en la Infancia - atribuye a las familias el papel de protagonistas en el proceso de apoyo a los niños, a través de su participación activa, considerando sus necesidades y preocupaciones. El propósito de este estudio es conocer cuáles son las prácticas que están siendo adoptadas por los profesionales a partir de las perspectivas de las familias apoyadas. Así, el objetivo del estudio se centra en identificar las prácticas actuais que se están implementando en el proceso de apoyo de Atención Temprana a nivel internacional. Con la formulación de criterios de selección, que se basaron en el año de publicación; tipo/naturaleza del estudio; objetivos y sujetos; se procedió a una investigación exhaustiva de la literatura publicada entre 2011 y 2017, existente en las bases de datos bon, ERIC, Scopus y Scielo. Después de la lectura de 1002 títulos de estudios, identificamos 79 estudios, de los cuales leemos el resumen y de éste pasamos a la lectura integral del estudio. Por lo que encontramos 14 estudios que cumplen los criterios de selección. El análisis se guía por las directrices para la implementación de las prácticas centradas en la familia y por lo tanto en cuatro categorías: (1) prácticas relacionales; (2) prácticas participativas; (3) calidad técnica del profesional; (4) equipo transdisciplinario. Estos estudios proporcionan evidencia de la importancia y utilidad de la Atención Temprana en la Infancia y de sus beneficios para los niños y sus familias. Los servicios prestados fueron positivamente útiles en la comprensión de las necesidades y competencias de las familias 
y de sus niños y contribuyeron a su desarrollo. Surgen contribuciones de indicadores de competencias profesionales.

Palabras clave: Atención temprana em la Infancia; Prácticas centradas em la família;

Participación.

\section{Introdução}

A abordagem centrada na família é um dos eixos principais das práticas recomendadas ao nível internacional para a Intervenção Precoce na Infância (IPI). Esta intervenção é caracterizada por práticas que envolvem um tratamento das famílias com dignidade e respeito, enfatizam os seus pontos fortes, compartilham informações para que possam tomar decisões informadas, fomentam oportunidades de escolha sobre os recursos necessários e desenvolvem uma relação de colaboração e parceria entre a família e o profissional de IPI (DUNST; TRIVETTE; HAMBY, 2007; ESPE-SHERWINDT, 2008). Estas práticas compreendem duas dimensões - a relacional e a participativa. As práticas relacionais incluem práticas associadas à escuta ativa, empatia e respeito. As participativas incluem práticas individualizadas, flexíveis e sensíveis às preocupações e prioridades das famílias, implicando o seu efetivo envolvimento em todas as situações que dizem respeito a todos os seus membros (DUNST et al., 2007). Daí o envolvimento das famílias ser fulcral na eficácia da prestação de intervenções de IPI como uma oportunidade de respeitar a sua singularidade e diversidade; bem como de reconhecer e compreender as sinergias contingentes com a resposta às necessidades e prioridades das famílias. Pelo que importa conhecer as perspectivas das famílias sobre as práticas de Intervenção Precoce na Infância, referenciadas na literatura internacional.

Estruturalmente, contextualizamos a IPI e a implementação da abordagem centrada na família. Seguidamente, descrevemos a metodologia; apresentamos e interpretamos os dados; e, por último, expomos as conclusões.

\section{Contextualização teórica}

A Intervenção Precoce na Infância (IPI), enquanto fonte de suporte a crianças com deficiência e/ou em risco de atraso de desenvolvimento (MCWILLIAM, 2010) e às suas famílias, compreende as experiências e oportunidades diárias, mediadas pelos pais e profissionais, para crianças (do nascimento aos 6 anos de idade), através de atividades de aprendizagem decorrentes em ambientes naturais, a fim de proporcionar a aquisição e prática de competências ajustadas às interações do seu quotidiano (DUNST et al, 2012). 
A IPI pretende, através da concretização de uma abordagem centrada na família (DUNST, 2002; ESPE-SHERWINDT, 2008) e baseada nas rotinas (MCWILLIAM, 2010), em contextos naturais de aprendizagem, realizada em equipa transdisciplinar, e assente em coordenação e integração de serviços (CARVALHO et al., 2016), contribuir para o fortalecimento do funcionamento familiar, condição para uma melhor qualidade de vida, incluindo o desenvolvimento e aprendizagem das crianças.

A dimensão participativa das famílias é a componente essencial da eficácia das práticas centradas na família, atribuindo às famílias o papel de protagonistas no processo de apoio às crianças.

A abordagem centrada na família distingue-se de outras abordagens por a família ser considerada capaz de tomar decisões informadas e pelas intervenções centrarem-se no fomento de competências e na mobilização de recursos e apoios para a família de uma forma individualizada, flexível e responsiva, segundo CARVALHO ET AL. (2016). De acordo com os autores, uma abordagem centrada na família é implementada através de: (i) práticas relacionais; (ii) práticas participativas; (iii) a qualidade técnica do profissional; e (iv) uma equipa com funcionamento transdisciplinar. Este modelo consiste num modo de funcionamento de uma equipa composta por profissionais de várias áreas (saúde, educação, etc.), que trabalham de uma forma colaborativa, cuja intervenção é prestada por um elemento da equipa - mediador de caso - que se encontra sempre apoiado pelos outros profissionais da equipa. Assim, a família não é sobrecarregada com visitas de diversos especialistas e pode estabecer mais facilmente uma relação de confiança com o mediador.

Assim, as famílias, como agentes de mudança e decisores finais, devem mostrar qual é o melhor caminho a percorrer na IPI. Portanto, a questão norteadora deste estudo é: Quais as práticas que estão a ser implementadas pelos profissionais de Intervenção Precoce na Infância segundo as perspectivas das famílias apoiadas?

\section{Metodologia}

O corpus compõe-se por 14 estudos empíricos que foram pesquisados e recolhidos no período de tempo entre novembro de 2016 a janeiro de 2017, nas bases de dados b-on, ERIC, Scopus e Scielo.

$\mathrm{Na}$ concretização desta revisão da literatura adotámos os procedimentos de uma revisão sistemática na seleção dos estudos, devido ao seu explicito rigor; e utilizámos a análise de conteúdo (AMADO, 2013; BARDIN, 2013) para analisar os resultados dos 


\section{http://dx.doi.org/10.5902/1984686X29789}

estudos com vista a encontrar a resposta à nossa questão de investigação. De acordo com BRYMAN (2012, p.103), para a consecução da presente revisão sitemática seguiu-se as etapas: (i) definir o propósito e o objetivo da revisão; (ii) pesquisar estudos relevantes segundo o propósito; (iii) verificar a adequação do estudo relativamente à questão de investigação; e (iv) analisar cada estudo e sintetizar os resultados.

\section{Procedimentos de investigação}

Conforme as indicações de Bryman (2012, p. 103), os procedimentos efetuados neste estudo consistiram na:

$1^{\circ}$ - Definição da questão de investigação;

$2^{\circ}$ - Formulação dos critérios de inclusão e de exclusão para a seleção de estudos empíricos;

$3^{\circ}$ - Realização de uma pesquisa abrangente de estudos nas bases de dados b-on, ERIC, Scopus e Scielo;

4ํ- - Aplicação dos critérios formulados; procedemos à avaliação dos estudos e selecionámos;

5ำ - Análise dos resultados de cada estudo.

\section{Critérios de seleção}

Os critérios de seleção formulados basearam-se em:
I. $\quad$ a data de publicação - seleção de estudos mais recentes;
II. o tipo de estudo e/ou sua natureza - seleção de estudos de natureza qualitativa/quantitativa/mista;
III. o problema/objetivo de investigação - práticas de Intervenção Precoce na Infância implementadas no apoio às famílias;
IV. os sujeitos de investigação - famílias/pais.

Considerámos pertinente definir um critério de exclusão de forma a rejeitar todos os estudos que não apresentassem uma metodologia e de excluir todos os estudos em que os participantes fossem profissionais. Se o problema ou objetivo de investigação não se encontrava claramente relacionado com a Intervenção Precoce na Infância, envolvendo crianças até aos seis anos de idade, levou à exclusão do estudo. A data de publicação compreendeu o período entre janeiro de 2011 até janeiro de 2017, de forma a incluir as investigações mais actualizadas. O idioma do artigo não constituiu um critério de inclusão nem de exclusão. 
http://dx.doi.org/10.5902/1984686X29789

\section{Processo da pesquisa e seleção dos estudos empíricos}

A escolha das palavras-chave foi efetuada a partir da questão de investigação. Assim, combinámos as palavras-chave de modo a que estas correspondessem, simultaneamente, ao nosso propósito: ("Early childhood intervention" OR "Early intervention") AND (family OR parents).

Com a inserção desta combinação nas bases de dados b-on, ERIC, Scopus e Scielo surgiram, no total, 202523 referências. Este número foi gradualmente reduzido à medida que os critérios de seleção foram aplicados. Desta forma, a restrição da data de publicação, entre 2011 e 2017, levou-nos a 79793 estudos. A seguir, este número foi reduzido pelo assunto/descritor/área resultando em 3658 estudos. Devido ao número ainda elevado, selecionamos pelo título (campo), unicamente, na base de dados b-on, obtendo 1002 estudos. Destes, voltámos a selecionar o assunto early intervention e obtivemos 561 estudos. Analisámos os títulos e, a seguir, lemos o resumo de cada estudo para perceber o tipo de estudo, o problema/objetivo da investigação e os seus participantes, pelo que obtivemos 79 estudos. Destes passámos para 15 estudos, através da sua leitura integral. Por último, avançámos com a avaliação dos estudos para percebermos se continha um enquadramento teórico explícito; a descrição clara da metodologia, dos resultados e das suas conclusões, conforme sugerido por Bryman (2012). Após esta avaliação, um estudo foi excluído devido aos seus resultados não incluírem informação sobre as práticas de IPI (MAS et al, 2016). Assim, o número final de estudos selecionados foi 14, em que oito resultaram da b-on (DUNST; BRUDER; ESPE-SHERWINDT, 2014; FORDHAM, GIBSON, BOWES, 2011; GAVIDIA-PAYNE; MEDDIS; MAHAR; 2015; GOLUBOVIC; MARKOVIC; PEROVIC; 2015; LEITE; PEREIRA, 2013; PIGHINI et al, 2014; ZHENG et al, 2016; ZIVIANI et al, 2014); três da ERIC (ALOTAIBI; ALMALKI, 2016; COOGLE; GUERETTE, HANLINE, 2013; THOMPSON; BRUNS, 2013); dois da Scopus (BAYHAN; SIPAL, 2011; BRANDÃO; CRAVEIRINHA, 2011); e um da Scielo (MAYORGA-FERNÁNDEZ; MADRID-VIVAR; GARCÍA-MARTíNEZ, 2015). As características dos estudos selecionados encontram-se apresentadas na tabela 1 (ver anexo).

Os estudos selecionados foram objeto de validação por uma especialista ${ }^{1}$ em IPI.

A figura 1 apresenta, de forma esquemática, o processo descrito.

\footnotetext{
1 Professora Paula Santos, Professora Auxiliar no Departamento de Educação e Psicologia da Universidade de Aveiro, Membro da direção da ANIP (Associação Nacional de Intervenção Precoce); Membro da EURLYAID - the European Association on Early Childhood Intervention.
} 
http://dx.doi.org/10.5902/1984686X29789

Figura 1 - Processo de seleção dos estudos empíricos

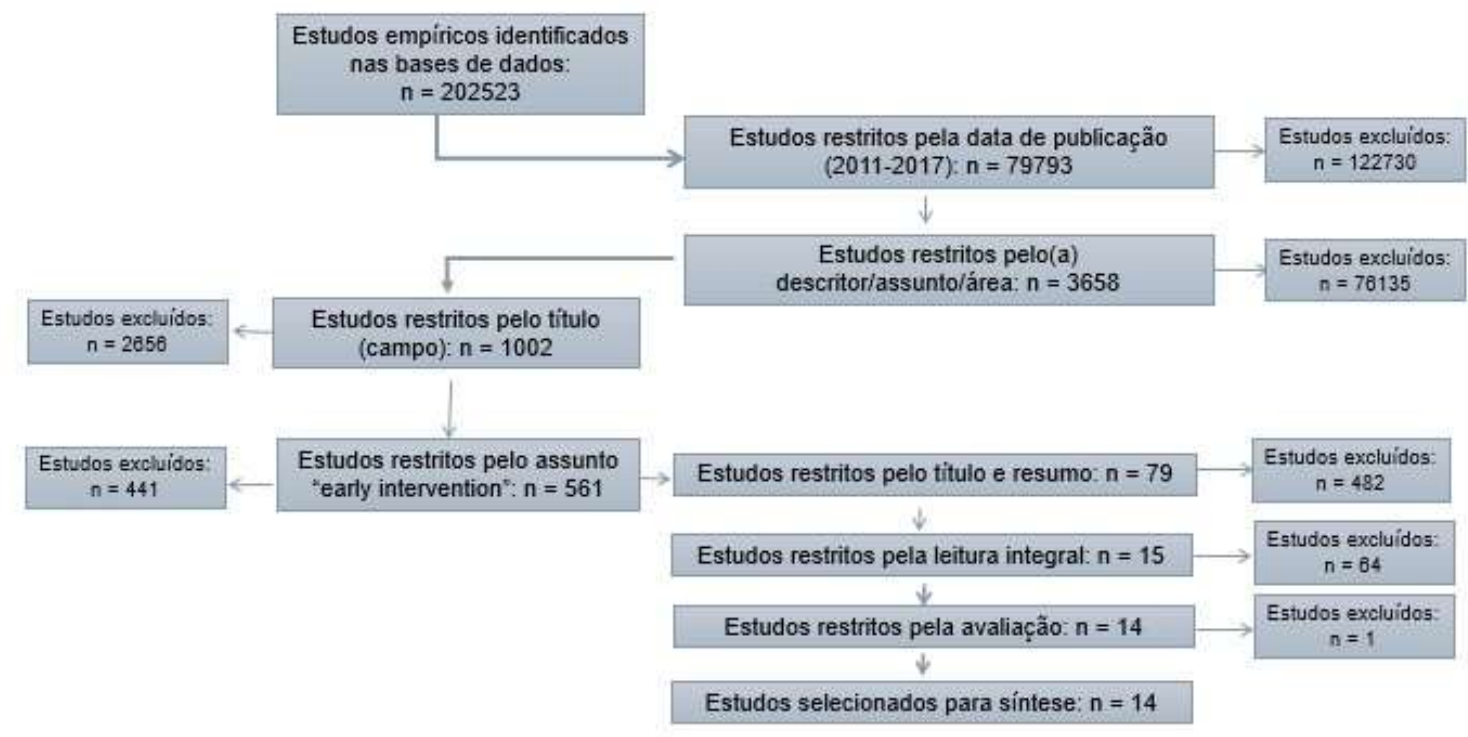

\section{Análise de conteúdo}

A análise de conteúdo foi selecionada para analisar os resultados dos estudos empíricos por permitir inferências interpretativas sobre as características das práticas de IPI, a partir das perspectivas das famílias. Assim, seguimos os procedimentos segundo Amado (2013) e Bardin (2013). Procurámos e recortámos uma parcela dos resultados de cada estudo com um sentido próprio, em função do nosso objetivo. Avançámos com o tratamento da informação através da codificação. Identificámos quatro categorias a priori (AMADO, 2013), provenientes das orientações para a implementação da abordagem centrada na família (CARVALHO et al., 2016), isto é, fForam consideradas como categorias: (I) práticas relacionais; (II) práticas participativas; (III) qualidade técnica do profissional de IPI; e (IV) equipa transdisciplinar. Este sistema foi validado pela especialista em IPI que, por sua vez, validou os estudos selecionados.

\section{Apresentação e interpretação de dados}

\section{Características metodológicas dos estudos empíricos}

Os 14 estudos selecionados são provenientes de nove países, dos quais três estudos da Austrália; três dos Estados Unidos da América; dois de Portugal; um de Espanha; um da República da Sérvia; um da Turquia; um do Canadá; um da Arábia Saudita e um da China. 
Todos os estudos envolveram famílias de crianças com idade até aos seis anos, apoiadas em IPI. A amostra total dos estudos foi 937 famílias.

Constatámos que a maioria dos participantes dos estudos foram as mães. Em dois estudos incluíram somente as mães; num estudo, surgiu como participantes, além do pai e/ou mãe, outro grau de parentesco não especificado e num estudo referenciou-se também a participação do avô.

A maioria dos estudos contemplaram as informações relativas aos participantes quanto ao sexo, à idade, ao estado civil, ao número de filhos, às habilitações académicas, ao rendimento mensal/anual, à situação profissional. No entanto, apenas em dois estudos (BRANDÃO; CRAVEIRINHA, 2011; GOLUBOVIC et al., 2015) há indicações da nacionalidade dos participantes e somente num estudo se encontra a raça/etnia dos sujeitos (THOMPSON; BRUNS, 2013).

A maioria dos autores selecionou opções metodológicas de natureza qualitativa. Esta evidência sustem o aumento do interesse e do uso da investigação qualitativa no campo da IPI mencionado por ERWIN, BROTHERSON E SUMMERS (2011).

Apesar das componentes culturais encontrarem-se ausentes, em praticamente todos os estudos, importa salientar que a diversidade familiar conduz à necessidade de uma compreensão do contexto de cada família e do desenvolvimento de intervenções em IPI, que se enquadrem nesses contextos ( $\mathrm{LYNCH}, 2013)$. Por isso, torna-se pertinente considerar as componentes culturais na elaboração dos instrumentos de recolha de dados, uma vez que as famílias compartilham muitas características, mas também diferem noutras, por isso, pode ser pertinente que a seleção da amostra contemple variáveis relacionadas com as dimensões da diversidade das famílias, os fatores socioculturais e as componentes culturais, como a língua materna, a raça/etnia, a religião.

\section{Apresentação e interpretação de dados obtidos a partir dos resultados dos estudos empíricos}

Os resultados serão apresentados tendo em consideração as categorias a priori provenientes das orientações para a implementação de uma abordagem centrada na família, isto é, (i) práticas relacionais; (ii) práticas participativas; (iii) qualidade técnica do profissional de IPI; e (iv) equipa transdisciplinar. 
(I) Práticas relacionais

As características inerentes às práticas relacionais foram salientadas em cinco estudos (COOGLE et al., 2013; PIGHINI et al., 2014; FORDHAM et al., 2011; ZHENG et al., 2016; ZIVIANI et al., 2014).

As características do profissional de IPI foram descritas em quatro estudos (COOGLE ET AL., 2013; PIGHINI ET AL., 2014; ZHENG ET AL., 2016; ZIVIANI ET AL., 2014). Segundo os seus resultados, as características interpessoais que as famílias participantes valorizaram no profissional incidiram no facto de ele ser organizado, amigo, compassivo, atencioso, útil, agradável e gentil. Por outro lado, de acordo com um estudo, uma família expressou que não estava satisfeita com os serviços e apoios de IPI devido, principalmente, ao desempenho dos profissionais por estes serem arrogantes, irresponsáveis (ZHENG ET AL., 2016).

A relação do profissional com a família foi identificada nos resultados de cinco estudos (COOGLE ET AL., 2013; PIGHINI ET AL., 2014; FORDHAM ET AL., 2011; ZHENG ET AL., 2016; ZIVIANI ET AL., 2014), em que as competências de comunicação na relação com as famílias basearam-se na confiança, no respeito, na compreensão, na escuta, na simpatia e na empatia. Esta última competência, foi referenciada num estudo, em falta, dado que uma família considerou que os profissionais não têm empatia com as crianças com deficiência (ZHENG ET AL., 2016).

Num estudo, os resultados revelaram que uma mãe explicou que a sua apreensão de ter alguém desconhecido a ir a sua casa deixou de existir quando a profissional explicouIhe que as visitas domiciliárias não necessitam de preparativos, além de que mais quatro mães, também expressaram o seu alívio em não ter que arrumar sempre a sua casa (PIGHINI ET AL., 2014). Esta evidência leva a sugerir que os primeiros contactos entre o profissional e a família são primordiais, uma vez que é quando se começa a estabelecer as bases de uma relação de confiança e de colaboração, ou seja, o que o profissional diz ou faz e a forma como escuta as famílias nos primeiros encontros vão constituir as primeiras impressões das famílias e influenciar os futuros contactos e fases da prestação de serviços de IPI. Portanto, esta fase inicial não deve ser substimada pelos profissionais, por isso deve-se respeitar o estilo de vida de cada família, escutar as suas prioridades, dar as informações necessárias e salientar que serão os pais a tomarem a decisão final no que respeita a si próprios, bem como às crianças. 
(II) Práticas participativas

Em todos os estudos, as práticas participativas encontram-se espelhadas nas perspectivas das famílias, conforme os resultados. São estas práticas que distinguem uma abordagem centrada na família de outro tipo de abordagens. A seguir apresentamos na tabela 1 os itens relevantes destas práticas encontrados nos estudos pesquisados, por ordem de maior frequência.

Tabela 1 - Itens das práticas participativas presentes nos estudos

\begin{tabular}{|l|c|l|}
\hline \multicolumn{1}{|c|}{$\begin{array}{c}\text { Itens das práticas } \\
\text { participativas }\end{array}$} & $\begin{array}{c}\text { No de } \\
\text { estudos }\end{array}$ & \multicolumn{1}{|c|}{ Referências } \\
\hline Redes de suporte das famílias & 9 & $\begin{array}{l}\text { NBAYHAN; SIPAL, 2011; BRANDÃO; CRAVEIRINHA, } \\
\text { 2011; COOGLE ET AL., 2013; FORDHAM ET AL., 2011; } \\
\text { LEITE; PEREIRA, 2013; MAYORGA-FERNÁNDEZ ET } \\
\text { AL., 2015; THOMPSON; BRUNS, 2013; ZHENG ET AL., } \\
\text { 2016; ZIVIANI ET AL., 2014 }\end{array}$ \\
\hline Participação & 6 & $\begin{array}{l}\text { BAYHAN, SIPAL, 2011; COOGLE et al., 2013; DUNST } \\
\text { et al., 2014; GAVIDIA-PAYNE et al., 2015; PIGHINI et } \\
\text { al., 2014; ZIVIANI et al., 2014 }\end{array}$ \\
\hline $\begin{array}{l}\text { Partilha de informação e de } \\
\text { materiais }\end{array}$ & 6 & $\begin{array}{l}\text { BAYHAN; SIPAL, 2011; COOGLE et al., 2013; } \\
\text { GOLUBOVIC et al., 2015; PIGHINI et al., 2014; } \\
\text { THOMPSON; BRUNS, 2013; ZIVIANI et al., 2014 }\end{array}$ \\
\hline Capacitação & 5 & $\begin{array}{l}\text { COOGLE et al., 2013; GOLUBOVIC et al., 2015; } \\
\text { PIGHINI et al., 2014; THOMPSON; BRUNS, 2013; } \\
\text { ZIVIANI et al., 2014 }\end{array}$ \\
\hline $\begin{array}{l}\text { Identificação de } \\
\text { necessidades/preocupações/pri } \\
\text { oridades }\end{array}$ & 3 & $\begin{array}{l}\text { COOGLE et al., 2013; PIGHINI et al., 2014; ZIVIANI et } \\
\text { al., 2014; }\end{array}$ \\
\hline $\begin{array}{l}\text { Tomada de decisão da família } \\
\text { em relação à criança }\end{array}$ & 2 & \begin{tabular}{l} 
PIGHINI et al., 2014; ZIVIANI et al., 2014 \\
\hline
\end{tabular} \\
\hline
\end{tabular}

As redes de suporte foram mencionadas em nove estudos (BAYHAN; SIPAL, 2011; BRANDÃO; CRAVEIRINHA, 2011; COOGLE ET AL., 2013; FORDHAM ET AL., 2011; LEITE; PEREIRA, 2013; MAYORGA-FERNÁNDEZ ET AL., 2015; THOMPSON, BRUNS, 2013; ZHENG ET AL., 2016; ZIVIANI ET AL., 2014), com maior incidência no apoio informal. Embora as fontes de apoio mais disponíveis se encontrem incluídas na rede formal de apoio social (BRANDÃO \& CRAVEIRINHA, 2011), segundo as perspectivas de famílias seria necessário existir uma associação de famílias para orientar e apoiar novas famílias com uma criança com deficiência, conforme o estudo de MAYORGA-FERNÁNDEZ ET AL. (2015). Por conseguinte, metade das famílias deste estudo relatou que os centros de IPI deveriam facilitar espaços para a compartilha e convivência entre famílias de crianças com deficiência, dado que uma grande percentagem de famílias considera importante conhecer os estilos de outras famílias, bem como expressar emoções e desabafos e, por isso, 0 relacionamento com outros pais pode ser considerado uma fonte valiosa de apoio e 
http://dx.doi.org/10.5902/1984686X29789

informação (ZIVIANI ET AL., 2014). Neste sentido, as perspectivas espelhadas nos resultados da maioria dos estudos sugerem que o associativismo é uma dimensão que pode ser muito relevante para o empowerment das famílias. Assim, a implementação de práticas centradas na família requer que se utilize todo o sistema de apoio da família. Consequentemente, importa que os profissionais tenham em consideração a mobilização de fontes de apoio informal (BRANDÃO \& CRAVEIRINHA, 2011), uma vez que podem ter uma maior duração temporal nas suas vidas, comparativamente com o apoio formal. Estas evidências encontram-se em consonância com os estudos de HIEBERT-MURPHY ET AL. (2011); SERRANO (2007) E SWAFFORD ET AL. (2015).

Além dos pais serem participantes ativos no apoio de IPI como sugerem os resultados do estudo de GAVIDIA-PAYNE ET AL. (2015), o estudo de PIGHINI ET AL. (2014) realça o relato de uma família em que a participação de um irmão mais velho nas sessões de IPI levou à sua capacitação no apoio à irmã, reforçando que os irmãos também são "professores" e acompanham o desenvolvimento de competências da criança de acordo com as rotinas diárias. Por conseguinte, devemos realçar a importância dos irmãos como agentes de socialização por excelência à criança e, por isso, torna-se obrigatório dar-lhes a devida importância no desenvolvimento da mesma (REIS; ESPE-SHERWINDT; SERRANO, 2010).

No estudo de PIGHINI ET AL. (2014) foi referido que as sessões em casa com um profissional de IPI não funcionou porque os membros da família não podiam participar. Por isso, é que tal como revelam os resultados do estudo de ZIVIANI ET AL. (2014), a maioria dos pais compreendem os programas como centrados na família, envolvendo todos os seus membros. Daí que, se os pais não são convidados a participar no apoio, como demonstram os resultados do estudo de BAYHAN E SIPAL (2011), parece que o conceito de práticas centradas na família está a ser mal-entendido, como corrobora o estudo de ESPESHERWINDT e SERRANO (2016).

Os resultados do estudo de DUNST ET AL. (2014) demonstraram que o local onde é prestada a intervenção de IPI influencia a probabilidade de os pais estarem ou não envolvidos na sua capacitação familiar. Isto é, as práticas de IPI prestadas, total ou parcialmente, nas casas das famílias foram relacionadas com um maior envolvimento delas. Os resultados deste estudo também indicam a necessidade de mais pesquisas para determinar quais os aspectos dos diferentes contextos que promovem ou impedem o envolvimento dos pais. Na verdade, os locais preferenciais para as práticas de IPI são os 
http://dx.doi.org/10.5902/1984686X29789

espaços onde seja possível apoiar o seu desenvolvimento. Portanto, segundo CARVALHO ET AL. (2016), o local deve ser escolhido a partir das necessidades das crianças e da família ou de outros cuidadores como a educadora de infância, a ama, os avós, tendo em consideração também os locais onde a criança passa mais tempo.

A partilha de informação e de materiais encontra-se descrita nos resultados de seis estudos (BAYHAN; SIPAL, 2011; COOGLE ET AL., 2013; GOLUBOVIC ET AL., 2015; PIGHINI ET AL., 2014; THOMPSON; BRUNS, 2013; ZIVIANI ET AL., 2014). A informação dada pelos profissionais foi considerada útil segundo os resultados dos estudos de GOLUBOVIC ET AL. (2015) e THOMPSON E BRUNS (2013), essencialmente para as famílias entenderem os comportamentos das suas crianças.

Todavia, é possível verificar nos resultados de dois estudos (BAYHAN; SIPAL, 2011; ZIVIANI ET AL., 2014) que há famílias que não obtiveram informações acerca os grupos sociais e recursos relativos à deficiência, como também expressaram insatisfação com a quantidade e qualidade da informação. Num destes estudos (BAYHAN; SIPAL, 2011), ainda é referido, por uma minoria, que não se encontrava informada sobre a opção da IPI ser realizada no contexto domiciliário. Pois, como já foi referido precedentemente, este contexto pode influenciar as perceções das famílias quanto ao apoio de IPI. Para uma intervenção centrada na família, é necessário que os pais tomem decisões logo desde os primeiros encontros, portanto é fulcral a disponibilização de informações. Por isso, poderá ser pertinente facultar um folheto informativo sobre o funcionamento quer da equipa, quer dos serviços existentes e de como são prestados.

A capacitação encontra-se descrita em cinco estudos (COOGLE ET AL., 2013; GOLUBOVIC ET AL., 2015; PIGHINI ET AL., 2014; THOMPSON; BRUNS, 2013; ZIVIANI ET AL., 2014), encontrando-se evidências nestes estudos de que os profissionais de IPI fornecem sugestões, instruções e direções e, por isso, os pais tornam-se mais competentes (PIGHINI ET AL., 2014), e sentem-se mais confiantes gradualmente (THOMPSON; BRUNS, 2013). Não obstante, o estudo de ZIVIANI ET AL. (2014), relata que um participante sentiu a falta de preparação para desempenhar o papel de pai de uma criança com deficiência. Esta evidência sugere que, por vezes, também é necessário ensinar os pais a ensinarem os seus filhos, quando as famílias querem saber o que fazer com os seus filhos. Uma das razões que podem levar a esta situação é o facto de a criança ter dificuldade em aprender ou a desempenhar uma atividade. Pois, ao contrário de crianças com desenvolvimento normal, cuja aprendidazem decorre, no geral, sob o incentivo dos pais; 


\section{http://dx.doi.org/10.5902/1984686X29789}

nestas crianças com dificuldades, a aprendizagem pode não decorrer tão facilmente, daí a necessidade sentida pelos pais de saber o que fazer para promover a aprendizagem da criança.

A identificação de necessidades / preocupações / prioridades foi narrada em três estudos (COOGLE ET AL., 2013; PIGHINI ET AL., 2014; ZIVIANI ET AL., 2014). Descobrir o que desejam as famílias, identificando as suas necessidades, preocupações e prioridades, de forma a compreender as famílias, possibilita intervenções mais individualizadas e mais eficazes.

Nos resultados de dois estudos encontra-se patente a tomada de decisão pela família (PIGHINI ET AL., 2014; ZIVIANI ET AL., 2014) quanto à criança, em que num destes estudos evidencia-se a relação entre a disponibilização de informação com a tomada de decisão, ou seja, alguns pais sentiram que tinham informações e apoio insuficientes para ajudá-los com a tomada de decisões em torno das opções escolares (ZIVIANI ET AL., 2014).

Como já foi mencionado acima, desde os primeiros contactos, com base na informação partilhada, é importante a família tomar decisões relativamente aos apoios de IPI.

\section{(III) Qualidade técnica do profissional de IPI}

Os resultados de seis estudos (ALOTAIBI; ALMALKI, 2016; COOGLE ET AL., 2013; PIGHINI ET AL., 2014; THOMPSON; BRUNS, 2013; ZHENG ET AL., 2016; ZIVIANI ET AL., 2014) expõem a qualidade técnica dos profissionais de IPI. Destes, três estudos (ALOTAIBI; ALMALKI, 2016; COOGLE ET AL., 2013; ZHENG ET AL., 2016) abordam a formação em IPI; um estudo (ZIVIANI et al., 2014) relata a experiência do profissional de IPI; e dois estudos (PIGHINI ET AL., 2014; THOMPSON; BRUNS, 2013) referem-se aos conhecimentos do profissional.

Segundo o estudo de COOGLE ET AL. (2013) a atuação dos profissionais de IPI não varia de acordo com as suas áreas profissionais. Enquanto no estudo de ALOTAIBI E ALMALKI (2016), as famílias afirmaram que o desenvolvimento profissional é uma exigência crucial para a prestação de serviços de IPI, um dado consonante com o estudo de HIEBERT-MURPHY, TRUTE E WRIGHT (2011).

No estudo de ZIVIANI ET AL. (2014) é relatado que, como um participante não recebeu as instruções necessárias sobre a implementação do apoio em casa, por isso, 
considerou os profissionais inexperientes. Por outro lado, no estudo de PIGHINI ET AL. (2014), todos os pais enfatizaram o aumento de conhecimentos e de uma profunda compreensão da condição da criança e do seu desenvolvimento. Já no estudo de THOMPSON e BRUNS (2013), um participante referiu que os profissionais de IPI forneceram detalhes técnicos sobre as quais ele não tinha conhecimento.

Estas evidências, em corroboração com o estudo de HIEBERT-MURPHY ET AL. (2011), sugerem a importância da formação contínua dos profissionais para que estejam constantemente actualizados e coerentes com as práticas recomendadas em IPI, dado que as necessidades mais incidentes destas famílias são as de conhecimento / informação, o que por sua vez, na falta deste conhecimento, tornar-se-á mais dificil a sua capacitação e a tomada de decisões (ZIVIANI ET AL., 2014).

\section{(IV) Equipa transdisciplinar}

Os resultados de dois estudos (FORDHAM ET AL., 2011; ZIVIANI ET AL., 2014) relataram o funcionamento da equipa transdisciplinar. Pelo que, as famílias que tiveram um mediador de caso referiram níveis mais elevados de apoio, segundo o estudo de FORDHAM ET AL. (2011). Nos resultados do estudo de ZIVIANI ET AL. (2014), além de as famílias recomendarem a colaboração dentro da equipa de IPI, também relataram que os profissionais tendiam a não manter contacto com outros serviços e que era, particularmente, preocupante a falta de comunicação entre as creches e as equipas de IPI. Pois, como corrobora CARVALHO ET AL., (2016), a colaboração é o motor do trabalho realizado em equipa transdisciplinar, pelo que é um aspeto que merece uma maior atenção.

É possível verificar que o funcionamento das equipas transdisciplinares foi reportado com menor incidência, em parte por ser um apoio de retaguarda, um avez que que não se torna tão visível, nem exposto nas perspectivas das famílias.

\section{Conclusão}

Para esta revisão analisou-se a metodologia e os resultados de 14 artigos publicados entre janeiro de 2011 e janeiro de 2017. Destes estudos, o objetivo de investigação mais proeminente baseou-se em conhecer as perceções das famílias apoiadas quanto às práticas de IPI. Assim, uns estudos identificaram perceções sobre os apoios que as famílias 
recebem e/ou as experiências com os profissionais; alguns estudos focaram os resultados e os benefícios das práticas de IPI e/ou da sua influência na vida das famílias; outros estudos abordaram a identificação das necessidades das famílias apoiadas em IPI; e um estudo focou as redes de apoio social das famílias.

Todos os estudos envolveram famílias como sujeitos de investigação e a maioria selecionou opções metodológicas de natureza qualitativa.

Os resultados dos estudos revelam perceções e experiências positivas das famílias em relação aos serviços prestados de IPI e que estes promovem o desenvolvimento das crianças. Em contraste, mas em menor dimensão, também encontrámos numa minoria de estudos (por exemplo, ZIVIANI ET AL., 2014), perceções distintas e sentimentos mistos, nas perspectivas das famílias, em relação ao apoio de IPI. Ambas as evidências corroboram as constatações reveladas pelo estudo de SWAFFORD ET AL. (2015). As perceções distintas das famílias devem-se, essencialmente, à insatisfação com a falta e / ou quantidade e qualidade de informações e orientações; à inexistência de oportunidades para a capacitação; e à não inclusão de membros da família na participação das sessões.

Embora se tenha reconhecido no estudo de BAYHAN e SIPAL (2011), que a implementação da abordagem centrada na família pode levar tempo, a análise dos resultados evidencia que as práticas relacionais e participativas funcionam, de um modo geral, consistentemente com a orientação teórica da abordagem centrada na família, em consonância com os resultados dos estudos de DUNST ET AL. (2007); HIEBERT-MURPHY ET AL., (2011) E SWAFFORD ET AL. (2015).

As práticas participativas - componente essencial da eficácia da abordagem centrada na família -, atribuindo às famílias o papel de protagonistas no processo de apoio às crianças - salientaram-se nas perspectivas das famílias, em que a participação e o envolvimento destas são primordiais em IPI.

É fundamental ter em consideração as oportunidades de desenvolvimento profissional (THOMPSON; BRUNS, 2013), pois este ajudará a melhorar as suas disposições, conhecimentos e competências no apoio às crianças e suas famílias (ZHENG ET AL., 2016), bem como a mobilização de fontes de apoio informal em vista da implementação de práticas centradas na família e culturalmente sensíveis (BRANDÃO; CRAVEIRINHA, 2011).

O apoio informal salientou-se nas perspectivas das famílias, no entanto, evidenciouse que ainda constituem um campo da IPI que precisa de se desenvolver e melhorar, uma vez que pode ter uma maior duração temporal nas suas vidas, comparativamente com o 
apoio formal. As associações e os grupos de pais podem ser uma via de apoio pertinente, como espaços de interação para as famílias, no enfrentamento das adversidades, pelo que o associativismo pode se constituir uma dimensão muito relevante para o empowerment das famílias.

Não foram encontradas evidências sobre as componentes culturais e a supervisão. No entanto a diversidade das famílias reflete-se nas práticas de IPI, com evidentes implicações para o profissional de IPI, no sentido de compreender e trabalhar respeitosamente com todo o tipo de famílias, através de práticas sensíveis às diferenças culturais, em que crenças, tradições, visões do mundo, língua, características físicas podem ser distintas das suas características (HANSON; LYNCH, 2012; LYNCH, 2013). Há também a considerar que a supervisão constitui uma dimensão essencial na procura de qualidade nas práticas de IPI (SANTOS, 2007). Portanto, é necessária atenção relativamente ao apoio aos profissionais e à sua capacitação, através da supervisão, para garantir a aplicação das competências desejadas e a verificação de marcadores apropriados para uma implementação de práticas centradas na família bem-sucedidas (HIEBERT-MURPHY et al., 2011).

Os resultados dos estudos refletiram (I) a relação do apoio dos profissionais de IPI com as experiências positivas de IPI; (II) a relação da disponibilização de informação com a capacitação familiar e o atendimento das necessidades das famílias: (III) a importância da participação de outros membros da família, essencialmente a dos irmãos mais velhos, nas sessões de apoio; (IV) a relação do contexto onde ocorre o apoio com o envolvimento das famílias; (V) a necessidade do fomento das redes informais de apoio; e (VI) a necessidade do desenvolvimento profissional em IPI.

Com base nas perspectivas das famílias, emergiram alguns indicadores passíveis de competências profissionais em IPI, como fortalecer a rede de suporte informal; apoiar as famílias de acordo com as práticas recomendadas ao nível internacional; dominar conhecimentos por forma a disponibilizar informação geral e específica; promover a participação e a capacitação das famílias.

De um modo geral, esta revisão evidenciou a importância e a necessidade da IPI na vida das famílias e das suas crianças. As pesquisas devem continuar a ouvir as vozes das famílias de forma a envolver as suas percepções e experiências, que vão influir as práticas de IPI, dado que as famílias são participantes essenciais na intervenção. 


\section{Referências}

ALOTAIBI, F.; ALMALKI, N. Parents' Perceptions of Early Interventions and Related Services for Children with Autism Spectrum Disorder in Saudi Arabia. International Education Studies, v. 9, n. 10, p. 128-140, 2016. doi: 10.5539/ies.v9n10p128

AMADO, J. Manual de Investigação Qualitativa em Educação. Coimbra: Imprensa da Universidade de Coimbra, 2013.

BARDIN, L. Análise de conteúdo. Lisboa: Edições 70, 2013.

BAYHAN, P.; SIPAL, R. F. Early intervention services in Turkey: Perspectives of southeastern families. International Social Work, v. 54, n. 6, p. 781-799, 2011. doi: 10.1177/0020872811404262

BRANDÃO, M. T.; CRAVEIRINHA, F. P. Redes de apoio social em famílias multiculturais, acompanhadas no âmbito da intervenção precoce: Um estudo exploratório. Análise Psicológica, v. 1, n. XXIX, p. 27-45, 2011.

BRYMAN, A. Social Research Methods. Oxford: Oxford University Press, 2012.

CARVALHO et al., Práticas recomendadas em Intervenção Precoce na infância. Um guia para profissionais. Coimbra: ANIP, 2016.

COOGLE, C. G.; GUERETTE, A. R.; HANLINE, M. F. Early Intervention Experiences of Families of Children with an Autism Spectrum Disorder: A Qualitative Pilot Study. Early Childhood Research \& Practice, v. 15, n. 1, 2013. Disponível em http://ecrp.uiuc.edu/v15n1/coogle.html

DUNST, C. J.; TRIVETTE, C. M.; HAMBY, D. W. Meta-Analysis of Family-Centered Helpgiving Practices Research. Mental Retardation and Developmental Disabilities Research Reviews, n. 13, p. 370-378, 2013.

DUNST, C. J.; RABB, M.; TRIVETTE, C. M.; SWANSON, J. Oportunidades de aprendizagem para a criança no quotidiano da comunidade. In MCWILLIAM, R. A., Trabalhar com as Famílias de Crianças com Necessidades Especiais, p.73-106, Porto: Porto Editora, 2012.

DUNST, C. J.; BRUDER, M. B.; ESPE-SHERWINDT, M. Family Capacity-Building in Early Childhood Intervention: Do Context and Setting Matter? School Community Journal, v. 24, n. 1, p. 37-48, 2014.

ERWIN, E. J.; BROTHERSON, M. J.; SUMMERS, J. A. Understanding Qualitative Metasynthesis: Issues and Opportunities in Early Childhood Intervention Research. Journal of Early Intervention, v. 33, n. 3, p. 186-200, 2011.

ESPE-SHERWINDT, M. Family-centred practice: collaboration, competency and evidence. Journal compilation, UK: Blackwell Publishing, 2008. 
ESPE-SHERWINDT, M, SERRANO, A. M. (2016). It takes two: The role of familycentered practices in communication intervention. Revista de Logopedia, Foniatría y Audiología, v. 36, p. 162-169.

FORDHAM, L.; GIBSON, F.; BOWES, J. Information and professional support: key factors in the provision of family-centred early childhood intervention services. Child: care, health and development, v. 38, n. 5, p. 647-653, 2011. doi:10.1111/j.13652214.2011.01324.x

FOX, S. E.; LEVITT, P.; NELSON, C. A. How the Timing and Quality of Early Experiences Influence the Development of Brain. Child Development, v. 81, n. 1, p. 28-40, 2010.

GAVIDIA-PAYNE, S.; MEDDIS, K.; MAHAR, N. Correlates of child and family outcomes in an Australian community based early childhood intervention program. Journal of Intellectual \& Developmental Disability, v. 40, n. 1, p. 57-67, 2015. Disponível em http://dx.doi.org/10.3109/13668250.2014.983056

GOLUBOVIC, S.; MARKOVIC. J.; PEROVIC, L. Things that can be changed in early intervention in childhood. Med Pregl, v. LXVIII, n. 7-8, p. 267-272, 2015. doi: 10.2298/MPNS1508267G

HANSON, M. J.; LYNCH, E. W. Trabalhar com famílias de meios sociais e culturais diferentes. In MCWILLIAM, R. A., Trabalhar com as Famílias de Crianças com Necessidades Especiais, p.165-196. Porto: Porto Editora, 2012.

HIEBERT-MURPHY, D.; TRUTE, B.; WRIGHT. Parents' Definition of Effective Child Disability Support Services: Implications for Implementing Family-Centered Practice. Journal of Family Social Work, v. 14, p. 44-158, 2011.

LEITE, C. S. C.; PEREIRA, A. P. S. Early intervention in Portugal: family support and benefits. Support for Learning, v. 28, n. 4, p. 146-153, 2013. doi: 10.1111/14679604.12034

LYNCH, E.W. Families in the 21st Century. In HANSON, M.J.; LYNCH, E.W., Understanding Families: Supportive Approaches to Diversity, Disability and Risk, pp.1-22. Baltimore: Brookes Publishing, 2013.

MAS, J. M. et al. Family Quality of Life for Families in Early Intervention in Spain. Journal of Early Intervention, v. 38, n. 1, p. 59-74, 2016 . doi: $10.1177 / 1053815116636885$

MAYORGA-FERNÁNDEZ, M. J.; MADRID-VIVAR, D.; GARCÍA-MARTÍNEZ, M. P. Aprender a trabajar con las familias en Atención Temprana: estudio de caso. Escritos de Psicología, v. 8, n. 2, 2015. Disponível em http://dx.doi.org/10.5231/psy.writ.2015.1306

MCWILLIAM, R. Routines-based Early Intervention. Supporting Young Children and Their Families. Baltimore: Paul H. Brookes Publishhing, 2010. 
PIGHINI, M. J. et al. Learning from parents' stories about what works in early intervention. International Journal of Psychology, v. 49, n. 4, p. 263-270, 2014. doi: 10.1002/ijop.12024

REIS, H.; ESPE-SHERWINDT, M.; SERRANO, A. M. O Perfil de Envolvimento e as Necessidades dos Irmãos das Crianças com Perturbação do Espectro do Autismo: Estudo descritivo realizado nos distritos do Porto, Viseu e Lisboa. INCLUSÃO, v. 10, p. 71-84, 2010.

SANTOS, P. (2007). Promovendo um processo de construção de uma cultura de Intervenção Precoce. Dissertação de doutoramento. Aveiro: Universidade de Aveiro. Disponível em http://hdl.handle.net/10773/1104

SERRANO, A. M. Redes Sociais de Apoio e Sua Relevância para a Intervenção Precoce. Porto: Porto Editora, 2007.

SHONKOFF, J. P. Building a New Biodevelopmental Framework to Guide the Future of Early Childhood Policy. Child Development, v. 1, n. 1, p. 357-367, 2010.

SWAFFORD, M. D. et al. Perceptions of Family-Centered Practices. Journal of Early Intervention, v. 37, n. 2, p. 138-154, 2015. doi: $10.1177 / 1053815115602880$

THOMPSON, S. D.; BRUNS, D. A. Perceptions of Early Intervention Services: Adolescent and Adult Mothers in Two States. Early Childhood Research \& Practice, v. 15, n. 1, 2013. Disponível em http://ecrp.uiuc.edu/v15n1/thompson.html

ZHENG, Y. et al. Early Childhood Intervention in China from the Families' Perspective. International Journal of Disability, Development and Education, v. 63, n. 4, p. 431449, 2016. doi: 10.1080/1034912X.2015.1124988

ZIVIANI, J. et al. Early intervention services of children with physical disabilities: Complexity of child and family needs. Australian Occupational Therapy Journal, v. 61, p. $67-75$, 2014. doi: 10.1111/1440-1630.12059

\section{Correspondência}

Marisa Alexandra Maia Machado - Universidade de Aveiro, Campus Universitário de Santiago, CEP: 3810-193, Aveiro, Portugal.

\section{(1) (8)}

This work is licensed under a Creative Commons Attribution-NonCommercial 4.0 International (CC BY-NC 4.0) 